\title{
Short geodesic segments between two points on a closed Riemannian manifold
}

\author{
Alexander Nabutovsky and Regina Rotman
}

June 25, 2008

\begin{abstract}
Let $M^{n}$ be a closed Riemannian manifold homotopy equivalent to the product of $S^{2}$ and an arbitrary $(n-2)$-dimensional manifold. In this paper we prove that given an arbitrary pair of points on $M^{n}$ there exist at least $k$ distinct geodesics of length at most $20 k ! d$ between these points for every positive integer $k$. Here $d$ denotes the diameter of $M^{n}$.
\end{abstract}

\section{Introduction and main results.}

In 1951 J.-P. Serre proved that for any pair of points on a closed Riemannian manifold $M^{n}$ there exist infinitely many geodesics connecting them. It is, therefore, natural to wonder about the lengths of these geodesics. For example, in [NR0] we made the following conjecture:

Conjecture A. There exists a function $f(k, n)$, such that for every closed Riemannian manifold $M^{n}$ of diameter $d$ and every pair of points $x, y \in M^{n}$ there exist at least $k$ geodesics between $x$ and $y$ of length at most $f(k, n) d$.

Example 1. For any two points on a round sphere there always exist $k$ geodesic segments of length $\leq k d$, (see fig. 1). On the other hand F.Balacheff, C. Croke and M. Katz ([BCK]) recently constructed examples of Riemannian metrics on $S^{2}$ arbitrarily close to the standard round metric such that the length of the shortest non-trivial geodesic loop based at every point is greater than $2 d$. These examples show that one cannot take $f(n, k)=k$ in Conjecture A even for $n=k=2$.

Example 2. Let $M^{n}$ be a closed Riemannian manifold with an infinite torsion-free fundamental group. Then it is not difficult to see that for any 


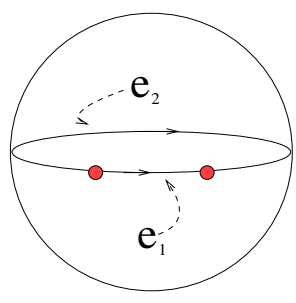

length $\left(e_{1}\right)$ is at most $d$, length $\left(\mathrm{e}_{2}\right)$ is at most $2 \mathrm{~d}$, $\mathrm{e}_{3}=\mathrm{e}_{1} *-\mathrm{e}_{2} * \mathrm{e}_{1}$ length $\left(\mathrm{e}_{3}\right)$ is at most $3 \mathrm{~d}$, $\mathrm{e}_{4}=\mathrm{e}_{2} *-\mathrm{e}_{1} * \mathrm{e}_{2}$ length $\left(e_{4}\right)$ is at most $4 d$, etc.

Figure 1: Short geodesic segments on a round sphere.

pair of points $p, q \in M^{n}$ there exist at least $k$ distinct geodesic segments of length $\leq k d$ ([NR0]). More generally, if $\pi_{1}\left(M^{n}\right)$ is either an infinite group or a finite group with at least $k$ elements one can consider the universal covering space of $M^{n}$ with the covering metric. Let $\tilde{p}$ be a lift of the base point in $M^{n}$ to the universal covering. It is known that one can choose the fundamental domain $U$ containing $\tilde{p}$ such that $U$ contains the set $S$ of all points $x$ of the universal covering for which $\tilde{p}$ is the closest to $x$ lifting of $p$, and $U$ is contained in the closure of $S$. As the distance from $\tilde{p}$ to any point of $S$ does not exceed $d$, the diameter of $U$ does not exceed $2 d$. Next consider a metric ball $B_{2 k d}(\tilde{p})$ centered at $\tilde{p}$ of radius $2 k d$. In that metric ball there exist at least $k$ fundamental domains isometric to $U$ and $k$ distinct points $\tilde{q}_{1}, \ldots, \tilde{q}_{k} \in\left\{\pi^{-1}(q)\right\}$. Consider $k$ minimal geodesic segments $\tilde{\alpha}_{i}, i=1, \ldots, k$, connecting $\tilde{p}$ with $\tilde{q}_{i}$. Each of them is projected onto a distinct geodesic segment $\alpha_{i}=\pi\left(\tilde{\alpha}_{i}\right)$ connecting the points $p$ and $q$ and of length $\leq d+2(k-1) d=(2 k-1) d$.

If the fundamental group of $M^{n}$ is finite and has cardinality $\leq k-1$, we can consider the universal covering $\tilde{M}^{n}$ of $M^{n}$. Its diameter will be at most $2(k-1) d$. If there exist $k$ distinct geodesic of length $\leq f(n, k) \operatorname{diam}\left(\tilde{M}^{n}\right)$ between every pair of points of $\tilde{M}^{n}$, then we can project these geodesics to $M^{n}$, obtaining at least $k$ distinct geodesics of length $\leq 2(k-1) f(n, k) d$ between eveery pair of points of $M^{n}$. Therefore the general case of Conjecture A would follow from its validity for simply connected manifolds.

Some other evidence of the validity of Conjecture A is our recent results [NR1] and [NR2] (see also [NR0]), where we showed that on every closed Riemannian manifold and for every pair of points of this manifold there exist at least two geodesics connecting these points of length $\leq 2 n d$. We also showed that if a Riemannian manifold $M$ is diffeomorphic to the 2sphere, then for every pair of points $x, y \in M$ and every $k$ there exist at 
least $k$ geodesic segments joining $x$ and $y$ of length at most $f(k) d$, where $f(k)=4 k^{2}-2 k-1$.

In this paper we will prove the following theorem.

Theorem 0.1 Let $M^{n}$ be a closed Riemannian manifold which is either homotopy equivalent to $S^{2} \times N^{n-2}$ for an arbitrary manifold $N^{n-2}$ or is a simply connected manifold such that for some map $\phi: S^{2} \longrightarrow M^{n}$ the composition $\phi \circ H$ of $\phi$ and the Hopf fibration $H: S^{3} \longrightarrow S^{2}$ represents a non-trivial element of infinite order in $\pi_{3}\left(M^{n}\right)$. Then for every pair of points $p, q \in M^{n}$ there exist at least $k$ distinct geodesics starting at $p$ and ending at $q$ of length $\leq 20 k ! d$. If $p=q$, then this upper bound can be replaced by a better upper bound $8 k ! d$.

Remark. Our calculations yield the upper bounds of the form $(8 k !+o(k !)) d$ for general $p, q$ and $(4 k !+o(k !)) d$ in the case, when $p=q$ in Theorem 0.1. As we do not believe that even these upper bounds are optimal, we decided to simplify the formulae for these upper bounds by choosing higher values of the constant factors at $k ! d$.

A nice proof of Serre's theorem can be found in a paper by Albert Schwarz ([Sch]). Schwarz also proved that the length of $k$ th geodesic can be majorized by $C\left(M^{n}\right) k$, where $C\left(M^{n}\right)$ does not depend on $k$ but only on the Riemannian manifold $M^{n}$. Here is a rough sketch of Schwarz's proof in the case when $M^{n}$ is simply connected. First, he makes an observation that the Cartan-Serre theorem (cf. [FHT], Theorem 16.10) implies that there exists an even-dimensional real cohomology class $u$ of the loop space $\Omega M^{n}$ such that all its cup powers $u^{i}$ are non-trivial. The space of paths $\Omega_{a, b}\left(M^{n}\right)$ is homotopy equivalent to $\Omega M^{n}$. An explicit homotopy equivalence $h: \Omega M^{n}=\Omega_{a, a}\left(M^{n}\right) \longrightarrow \Omega_{a, b} M^{n}$ can be constructed as follows: Fix a minimizing geodesic between $a$ and $b$. Attach this geodesic at the end of each loop based at $a$.

Now one can apply the Morse theory to produce critical points of the length functional on $\Omega_{a, b} M^{n}$ corresponding to cohomology classes $h^{*}\left(u^{i}\right)$ for $i=1,2, \ldots$. As $u^{i}=u^{j} \cup u^{i-j}$, when $i>j$, the standard LyusternikSchnirelman trick (cf. [Kl]) implies that either $h^{*}\left(u^{i}\right)$ and $h^{*}\left(u^{j}\right)$ correspond to different critical points (i.e. to distinct geodesics between $a$ and $b$ ) or the critical level corresponding to $h^{*}\left(u^{j}\right)$ contains a set of critical points of positive dimension. (In the last case there exists an infinite set of geodesics 
between $a$ and $b$ of the same length). So, the set of geodesics between $a$ and $b$ is infinite. In order to estimate their lengths it is sufficient to consider the case when $h^{*}\left(u^{i}\right)$ and $h^{*}\left(u^{j}\right)$ correspond to different critical points whenever $i \neq j$.

Now recall that the Pontryagin product in rational homology group of the loop space is the product induced by the geometric product $\Omega M^{n} \times \Omega M^{n} \longrightarrow$ $\Omega M^{n}$. (By the geometric product of two loops $\alpha$ and $\beta$ we just mean their join $\alpha * \beta$.) To estimate the length of the geodesics corresponding to $u^{i}$ Schwarz defines a "dual" real homology class $c$ of $u$ of the same dimension. ("Dual" means here that $\langle u, c\rangle=1$.) Then he proves that for every positive $i$ the $i$ th Pontryagin power of $c$ and a real multiple of $u^{i}$ are dual. So, the critical point corresponding to $u^{i}$ also corresponds to $c^{i}$. Choose a representative of $c$. Let $L$ be such that this representative is contained in the set of loops of length $\leq L$. Then $c^{i}$ can be represented by a chain contained in the set of loops of length $\leq i L$, and $h_{*}\left(u^{i}\right)$ can be represented by a chain contained in the set of paths of length $\leq i L+2 d$ between $a$ and $b$, whence the length of the $i$ th shortest geodesic between $a$ and $b$ does not exceed $i L+2 d$.

Note that although Schwarz's result indicates that it is natural to look for an upper bound of the form $c(n) k d$ for the length of the $k$ th shortest geodesic between $a$ and $b$, it does not imply that such an upper bound exists, and, in fact, the factorial behaviour in $k$ of our upper bound might be optimal.

Let $H: S^{3} \longrightarrow S^{2}$ denote the Hopf fibration. Assume that $M^{n}$ is simplyconnected and there exists a map $\phi: S^{2} \longrightarrow M^{n}$ such that $\phi \circ H: S^{3} \longrightarrow M^{n}$ represents a non-trivial class of infinite order in $\pi_{3}\left(M^{n}\right)$. Note that the Hopf fibration corresponds to a non-trivial element $\chi$ of $\pi_{2}\left(\Omega S^{2}\right)$, and the image $\mu$ of $\chi$ under the Hurewicz homomorphism $\pi_{2}\left(\Omega S^{2}\right) \longrightarrow H_{2}\left(\Omega S^{2}, R\right)$ is nontrivial. Our assumption about $\phi$ implies that $(\Omega \phi)_{*}$ maps $\chi$ into a nontrivial homotopy class of infinite order in $\pi_{2}\left(\Omega M^{n}\right)$. Now the Cartan-Serre theorem implies that this element corresponds to a two-dimensional real cohomology class $\nu$ of $\Omega M^{n}$ such that all its cup powers are non-trivial, and any integer multiple of $\nu$ has the same property and can be used instead of $u$ in the proof of the existence of an infinite set of geodesics between $a$ and $b$. We can find a dual two-dimensional homology class $c$ of $u$ as the image of $\mu$ under the homomorphism induced by $\Omega \phi$.

The class $\mu \in H_{2}\left(\Omega S^{2}, R\right)$ admits the following explicit description. Consider the 1-dimensional real homology class $\varrho$ of $\Omega S^{2}$ defined as the image of the fundamental homology class of $S^{1}$ under a map $\omega: S^{1} \longrightarrow \Omega S^{2}$ cor- 
responding to the identity map $S^{2} \longrightarrow S^{2}$. The map $\omega$ admits the following explicit geometric description: For every $\alpha \in S^{1} \omega(\alpha)$ is the loop based at the South pole that first goes to the North pole via the meridian with longitude $\alpha$ and the returns to to the South pole via the meridian with longitude zero. The class $\mu$ is equal to the Pontryagin square of $\varrho$ multiplied by two (cf. [FHT], pp. 234-235). Thus, $\mu$ can be represented by a singular chain in $\Omega S^{2}$ such that each loop in the image of each singular simplex in this chain consists of four meridians of $S^{2}$. In [NR2] we represented $\mu$ by another explicit chain so that the loops in the image of each singular simplex consisted of either two meridians of $S^{2}$ or a subset of a meridian of $S^{2}$ travelled in the opposite directions.

Thus, if there exists $\phi: S^{2} \longrightarrow M^{n}$ such that $[\phi \circ H] \in \pi_{3}\left(M^{n}\right)$ is an element of infinite order, then for every $k$ there exist $k$ different geodesics between $a$ and $b$ of length $\leq 2 k L+2 d$, where $L$ is the supremum of lengths of images of meridians of $S^{2}$ under $\phi$. (More precisely, by "the length of the image of a meridian of $S^{2}$ " under $\phi$ we mean here and below the measure of this meridian under the pullback measure induced by $\phi$. In other words, if the image of the meridian backtracks over itself, we count the length of the backtracking piece twice.) If $\phi$ maps the North pole or the South pole of $S^{2}$ to $a$ or to $b$, then the estimate can be improved to $2 k L+d$. If, in addition, $a=b$, then the upper bound becomes $2 k L$, as we do not need to use the homotopy equivalence $\Omega_{a, a} M^{n} \longrightarrow \Omega_{a, b} M^{n}$. (However, one of the $k$ distinct geodesic loops in this case will be trivial.)

Thus, we obtain the following proposition:

Proposition A. Let $M^{n}$ be a simply connected Riemannian manifold of diameter d. Assume that there exists a map $\phi: S^{2} \longrightarrow M^{n}$ such that the composition $\phi \circ H$ of $\phi$ and the Hopf fibration $H: S^{3} \longrightarrow S^{2}$ represents an element of infinite order in $\pi_{3}\left(M^{n}\right)$. Assume, further, that the length of the image of every meridian of $S^{2}$ under $\phi$ does not exceed $L$. Then for every two points $a, b$ and for every $k$ there exist at least $k$ distinct geodesics between $a$ and $b$ of length $\leq 2 k L+2 d$. If a coincides with the image of the South pole of $S^{2}$ under $\phi$, then the lengths of $k$ distinct geodesics between $a$ and $b$ do not exceed $2 k L+d$. In this case there exist at least $k$ distinct geodesic loops based at a of length $\leq 2 k L$.

Note, that essentially the same argument will be true for some non-simply connected manifolds. For example, it will be true for manifolds homotopy equivalent to $S^{2} \times N^{n-2}$, where $N^{n-2}$ is an arbitrary closed manifold. In 
this case one can define $\phi$ as the composition of an inclusion $S^{2} \longrightarrow S^{2} \times$ $N^{n-2}$ that sends $S^{2}$ into $S^{2} \times\{p t\}$ for some $p t \in N^{n-2}$ and the homotopy equivalence $S^{2} \times N^{n-2} \longrightarrow M^{n}$. (Of course, as we have already mentioned above, one can always reduce the case of non-simply connected manifolds to the simply-connected case paying the price of mutiplying the upper bound by $2(k-1)$. Here we are saying that if our manifold is homotopy equivalent to $S^{2} \times N^{n-2}$, then we will not need to multiply the upper bound that we are going to derive below by $2(k-1)$ even if $N^{n-2}$ is not simply-connected.)

Note that even if $M^{n}$ is diffeomorphic to $S^{2}$ one cannot hope to find a map $\phi: S^{2} \longrightarrow M^{2}$ of non-zero degree such that the images of all meridians are bounded by const $d$ for some constant const. It seems that a counterexample can be constructed by attaching to 2-discs constructed in [FK] any fixed 2-disc thus obtaining a family of 2 -spheres. But we have not checked the details.

Therefore we cannot hope to majorize the length of $k$ shortest geodesics by merely finding an appropriate map $\phi$ such that the lengths of images of meridians of $S^{2}$ under $\phi$ are controlled in terms of $d$. Our paper [NR] dealt with the case when $M^{n}$ was diffeomorphic to $S^{2}$. Our strategy there was to try to construct $\phi$ with a control over lengths of images of meridians so that our attempt could be thwarted only by an obstructing geodesic between $a$ and $b$. Then we were making another attempt of constructing a map $\phi$ so that the lengths of the images of meridians were bounded by a larger constant. The second attempt could be blocked only by a different geodesic between $a$ and $b$, and so on. After $k$ attempts we would either obtain $k$ distinct obstructing geodesics between $a$ and $b$ or a desired $\phi$ with a control over the length of images of meridians. (Of course, in the last case we would immediately obtain the desired upper bound for an infinite set of distinct geodesics connecting $a$ and $b$ ).

Our strategy of constructing $\phi$ in [NR2] was based on the twodimensionality of the manifold, and cannot be used in the situation of the present paper. Instead, we adopt a more complicated startegy.

Our general strategy will be to start with a map $\phi$ satisfying the condition of a proposition. We would be trying to establish a control over the length of the images of meridians not under $\phi$ but under the composition of $\phi$ with a map $\phi_{s}$ of $S^{2}$ into itself of degree $s$. The number $s$ grows from an attempt to an attempt. Again, eventually we will either get $k$ geodesics between $a$ and $b$ as obstructions, or will succeed in finding a self-map of $S^{2} \phi_{s}$ of degree $s=(k-1)$ ! such that the length of the images of meridians under $\phi \circ \phi_{s}$ is controlled. 
Let us make the following obvious remark: Consider a slicing (= a partition) of $S^{2}$ into circles emananting from the South pole. If one manages to establish a control over lengths of images of the circles under a map $\tau$, then one can establish a control over lengths of images of meridians of the map $\tau \circ \lambda$, where $\lambda$ is a self-map of $S^{2}$ of degree one that sends one meridian to the South pole and sends all meridians to the circles from the slicing.

Therefore, in practice we will be trying to sweep-out various non-trivial spherical 2-cycles in $M^{n}$ of interest for us by loops of controlled length. The existence of such a sweep-out for $\phi \circ \phi_{s}$ would imply that $\phi \circ \phi_{s} \circ \lambda$ maps meridians into loops of controlled length emanating from the same point.

The next section contains further ideas of the proof of the main theorem.

\section{Further ideas of the proof of main theorem.}

Here we are going to briefly describe some key elements of the proof as well as the proof in a very simple case. For simplicity of the exposition we assume that $M^{n}$ is homotopy equivalent to $S^{2} \times N^{n-2}$. (This assumption is not really necessary, but, in our opinion, it makes the exposition more transparent.)

For simplicity of an exposition, let us, first, deal with geodesic loops, which can be regarded as geodesic segments, in which the two end-points are the same. Note that a related problem of estimating the length of a shortest geodesic loop on a closed Riemannian manifold was first studied by $\mathrm{S}$. Sabourau in $[\mathrm{S}]$. (Sabourau did not consider the base point of a geodesic loop as being fixed and minimized over the set of all base points as well.) The upper bound 2nd for the length of the shortest non-trivial geodesic loop at every prescribed point was first proven in $[R]$ for an arbitrary closed $n$-dimensional manifold.

1. Obstruction argument. Let us begin with a homotopy equivalence $F: S^{2} \times N^{n-2} \longrightarrow M^{n}$. Consider $f=\left.F\right|_{S^{2} \times\{p t\}}$ for any point $p t \in N^{n-2}$. Obviously, $f$ is noncontractible, and, moreover, $f \circ H$ represents an element of infinite order in $\pi_{3}\left(M^{n}\right)$. (Recall that $H$ denotes the Hopf fibration.) By virtue of the discussion in the previous section, it would be enough to sweep-out $S^{2}$ by curves connecting a fixed pair of points (in particular, loops emanating from the same point), so that the lengths of their images under $f$ are controlled in terms of $d$. However, as we have no information about the geometry of $f$, this is not possible. The next objective, therefore, is to obtain a new map $g: S^{2} \longrightarrow M^{n}$, that can be swept-out by "short" curves, 
and, which belongs to the same homotopy class as the original map $f$.

Let us begin by endowing $S^{2}$ with a fine triangulation. We will try to extend $f: S^{2} \longrightarrow M^{n}$ to a 3 -dimensional disc.

As an obstruction to this extension we hope to obtain a noncontractible map $g: S^{2} \longrightarrow M^{n}$, naturally endowed with a sweep-out by short curves. The use of a similar obstruction technique for the purpose of obtaining stationary objects was originated by M. Gromov in $[\mathrm{G}]$. The technique that we will use in this paper is, however, significantly modified.

2. The extension procedure. Let $D^{3}$ be triangulated as a cone over $S^{2}$. The extension will be inductive to skeleta of $D^{3}$. Let us begin with the center of the disc $\tilde{p}$ that will be mapped to a point $p$ of the manifold. We will extend to the 1-skeleton, by mapping edges of the form $\left[\tilde{p}, \tilde{v}_{i}\right]$ to corresponding minimal geodesic segments of the form $\left[p, v_{i}\right]$, where $v_{i}=$ $f\left(\tilde{v}_{i}\right)$. Next let us extend to the 2-skeleton. Let us consider an arbitrary 2 -simplex $\left[\tilde{p}, \tilde{v}_{i}, \tilde{v}_{j}\right]$. Its boundary is mapped to a closed curve of length $\leq 2 d+\delta$. A length shortening process being applied to any curve with a fixed point $p$ either stops at a geodesic loop of a shorter length or contracts the curve to the point $p$. Let us apply the Birkhoff Curve Shortening Process (BCSP) for loops with fixed basepoint to the image of the boundary of the above simplex, (for the detailed description of the BCSP see $[\mathrm{C}]$ ). Assume that when we apply the BCSP to this curve with a fixed point $p$, it is not terminated by a geodesic loop. Then the curve is contracted to a point $p$ as a loop, and we can map the 2-simplex to a surface generated by this homotopy. Let us now look at the next skeleton. We claim that there are 3 -simplices in the 3 -skeleton of $D^{3}$, such that, the restrictions of the map $f$ on their boundaries are non-contractible, and the corresponding elements of $\pi_{2}\left(M^{n}\right)$ sum to the element represented by $f$. Also, by virtue of Lemma 2.1 below the boundaries of these 3 -simplices can be swept-out in a natural way by curves of length $\leq 3 d+\delta$, for some small $\delta$ that depends on the size of the triangulation. Therefore, it follows that at each point $p \in M^{n}$ there exist at least $k$ geodesic loops of length bounded by $k(6 d+2 \delta)$. We will eventually let $\delta$ approach 0 , and obtain the assertion of the theorem.

3. How to extend to the 2-skeleton in the presence of "short" geodesic loops. Of course, the assumption made in order to extend to the 2-skeleton is rarely satisfied. In general, we cannot hope that we can extend to the 2-skeleton as above, because the curves can get stuck on geodesic loops of length $\leq 2 d+\delta$. In fact, even one "small" geodesic loop can obstruct the extension, and makes it unclear how to continue. Therefore, let us look again at extension to the 1-skeleton and try to modify that as 
follows: Consider a simplex $\left[\tilde{p}, \tilde{v}_{i_{1}}, \tilde{v}_{i_{2}}\right]$. Its boundary is mapped to a closed curve $\left[v_{i_{1}}, v_{i_{2}}\right] *\left[v_{i_{2}}, p\right] *\left[p, v_{i_{1}}\right]$, (see fig. $2(\mathrm{a})$ ). Let us denote the edges that connect $p$ with $v_{i_{j}}$ by $e_{j}$ and the edges that connect $\tilde{p}$ with $\tilde{v}_{i_{j}}$ by $\tilde{e}_{j}$ respectively, where $j=1,2$. Next suppose the curve $e_{1} *\left[v_{i_{1}}, v_{i_{2}}\right] *-e_{2}$ gets stuck on a geodesic loop $l_{i_{1}, i_{2}}$ of length $\leq 2 d+\delta$. We will change our extension to 1-skeleton as follows: the extension will stay the same on $\tilde{e}_{1}$, but on $\tilde{e}_{2}$ it will become $l_{i_{1}, i_{2}} * e_{2}$. Now, indeed, we can extend to the 2 -simplex $\left[\tilde{p}, \tilde{v}_{i_{1}}, \tilde{v}_{i_{2}}\right]$, as the curve $e_{1} *\left[v_{i_{1}}, v_{i_{2}}\right] *-e_{2} *-l_{i_{1}, i_{2}}$ is homotopic to the point $p$, (see fig. 2 (b)). We will map the 2 -simplex to the disc generated by this homotopy. Now, if we only had to extend to this one 2 -simplex, we would be done, but, of course, in general it is impossible to extend to the whole 2 -skeleton in this way for the following reason:

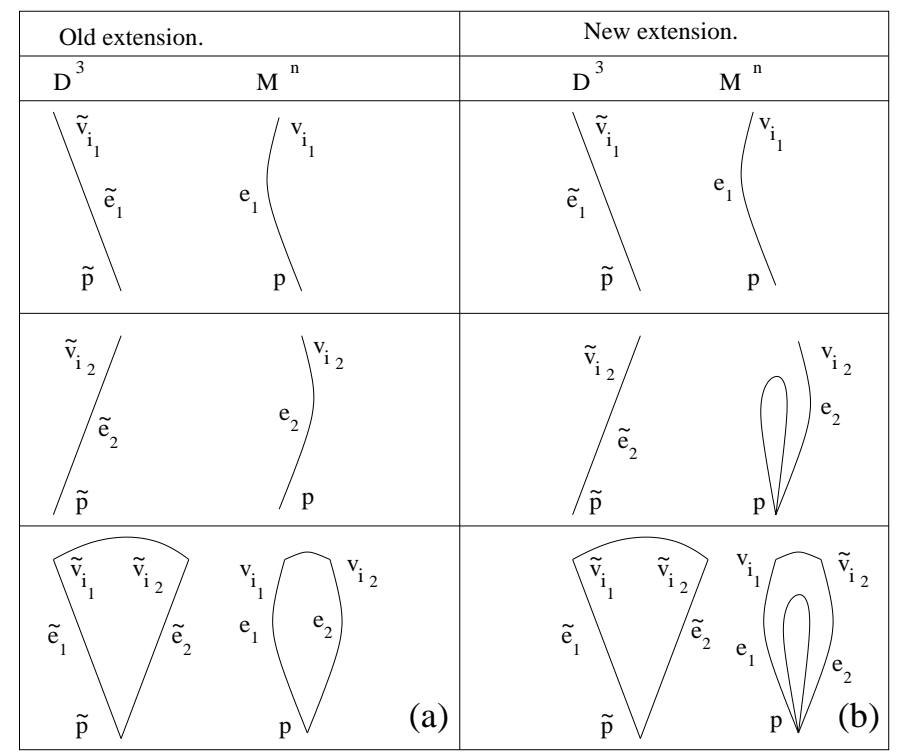

Figure 2: Modified extension.

Consider a simple 1-cycle in the 1-skeleton of the triangulation of $S^{2}$, (see fig. 3).

Let us denote the vertices of this graph by $\tilde{v}_{i_{1}}, \tilde{v}_{i_{2}}, \ldots, \tilde{v}_{i_{k}}$, let $v_{i_{j}}=$ $f\left(\tilde{v}_{i_{j}}\right), j=1, \ldots, k$. Next, let us denote the segments that connect $\tilde{p}$ with $\tilde{v}_{i_{j}}$ and $p$ with $v_{i_{j}}$ by $\tilde{e}_{j}$ and $e_{j}$ respectively. Suppose, we want to change the extension on 1-skeleton along this cycle, in such a way that will enable us to extend to 2 -skeleton. Let us begin with the 2 -simplex $\left[\tilde{p}, \tilde{v}_{i_{1}}, \tilde{v}_{i_{2}}\right]$. Sup- 

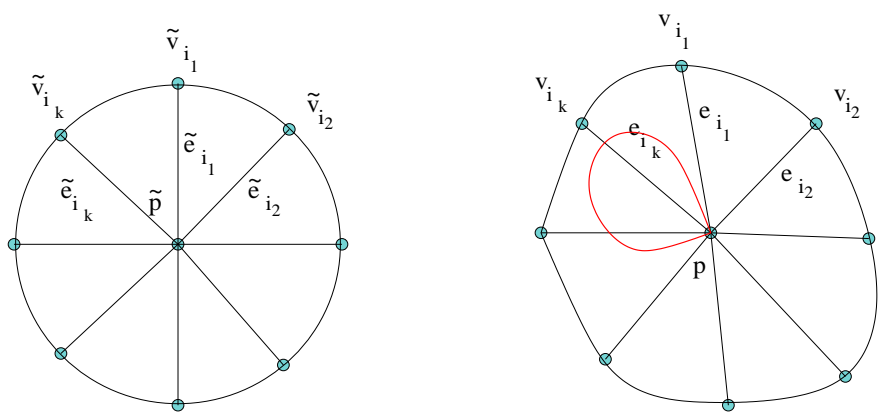

Figure 3: Why we cannot always extend.

pose that the image of the boundary of this simplex contracts to a geodesic loop $l_{i_{1}, i_{2}}$. We then modify an extension, so that $\tilde{e}_{2}$ is mapped to $l_{i_{1}, i_{2}} * e_{2}$. Next, let us consider $\left[\tilde{p}, \tilde{v}_{i_{2}}, \tilde{v}_{i_{3}}\right]$. Its boundary is mapped to a closed curve $l_{i_{1}, i_{2}} * e_{2} *\left[v_{i_{2}}, v_{i_{3}}\right] *-e_{3}$. Suppose that when we try to contract this curve it gets stuck on the same geodesic loop $l_{i_{1}, i_{2}}$. Then, we have to change an extension on $\tilde{e_{3}}$ to be mapped to $l_{i_{1}, i_{2}} * e_{3}$. It can so happen, that when we continue in such a way, we will have to change an extension on $\tilde{e_{k}}$ and next on $\tilde{e_{1}}, \tilde{e_{2}}$ and so, this process will not terminate, and the desired extension to the 2-skeleton will still be prevented by a single loop.

4. Partial modified extension. It is, thus, clear that the idea described in (1) cannot always work, but we would like to get something out of it. That is, if we cannot construct an extension to the whole 2-skeleton in such a way, we would at least try to extend it to a part of it. In fact, we would like to extend to at least one face of every 3 -simplex in the 3 skeleton of $D^{3}$. (Our reasons for doing so will become apparent at the end of section 2; cf. Remark 2.4.) It is not difficult to see that we can use the idea in the previous section to extend our map to the cone over any tree in the 1-skeleton of a considered triangulation of $S^{2}$ (after an appropriate modification of the already constructed extension to the 1-skeleton of this cone). So, the following lemma will be helpful for our purposes:

Lemma 1.1 Given $\delta>0$, there exists a triangulation of the 2-sphere, such that each simplex of the triangulation has a diameter $<\delta$ and satisfies the following property: There exist two nonintersecting trees in the 1-skeleton of the triangulation, such that any 2-simplex has an edge in one of the trees.

Proof. A triangulation satisfying the required properties will be constructed from the standard triangulation of the round 2- sphere as the boundary of a 
3 -simplex by successively performing any necessary number of operations of one of three types described below. It will be clear from the description of these operations that we can use an appropriate sequence of such operations to make the diameter of any 2-simplex less than $\delta$ for any positive $\delta$.

Let $a, b, c, d$ be the vertices of the standard triangulation of the boundary of a 3-simplex. In such a triangulation there exist two trees denoted $T_{1}$ and $T_{2}$ satisfying the conclusion of the Lemma . We can simply let $T_{1}$ consist of one edge $[b, d]$ and $T_{2}$ consist also of one edge $[a, c]$, (see fig. 5 (a)). Note also that each 2-simplex of this triangulation satisfies the following property. Opposite vertex property: The vertex of a simplex that is opposite to the edge that belongs to one of the trees, is in the different tree.

To make this triangulation finer, but still satisfying the same properties we can perform the following three operations:

(1) Subdivide an edge of a tree into two equal segments and join the middle with the opposite vertex in each of two 2-simplices adjacent to the edge, (see fig. $5(\mathrm{~b}))$. In this case, obviously, the triangulation satisfies the required properties, because the subdivided edge of the tree simply becomes two edges instead. The opposite vertex property will also not be violated by this operation.

To subdivide further, we will need to simultaneously subdivide pairs of simplices that share an edge which is not in one of the trees. There are two possibilities: either these simplices contain two edges that belong to the same tree as in fig. 4 (a), in which case the edges will share a vertex, or they contain two edges that belong to different trees, as in fig. 4 (b), in that case edges will be "opposite". This observation follows from the opposite vertex property. These two cases will be discussed separately and result in two different subdivisions.

(2) Consider two 2-simplices with a common edge not in one of the trees, like depicted on fig. 4 (a). Let us denote them $[a, c, x]$ and $[c, x, d]$ respectively. Suppose edges $[a, x]$ and $[x, d]$ are both in $T_{1}$ and the vertex $c$ is in $T_{2}$. Then we can subdivide the common edge $[c, x]$ into two equal segments, connect the middle with vertices $a$ and $d$, and add the edge obtained by subdividing $[c, x]$ and containing $c$ to $T_{2}$, (see also fig. $5(\mathrm{c})$ ).

(3) Consider two 2-simplices with a common edge not in one of the trees, like depicted on fig. 4 (b). Let us denote them as $[a, x, d]$ and $[a, y, d]$ respectively. Suppose now that $[y, d]$ is an edge in $T_{1}$ and $[a, x]$ is an edge in $T_{2}$. We can subdivide the common edge into three equal segments. Denote the points of subdivision by $w, z$ respectively, connect vertices $x, y$ with $w, z$ and add the edge $[y, w]$ to $T_{1}$ and the edge $[x, z]$ to $T_{2}$, (see fig. 4 (b) as 
well as fig. $5(\mathrm{~d}))$.

Note that none of the original specified properties of the triangulation including the opposite vertex property are violated by these operations.

Performing the three operations allows one to make the diameter of simplicies of a triangulation as small as desired, while the desired properties remain satisfied.

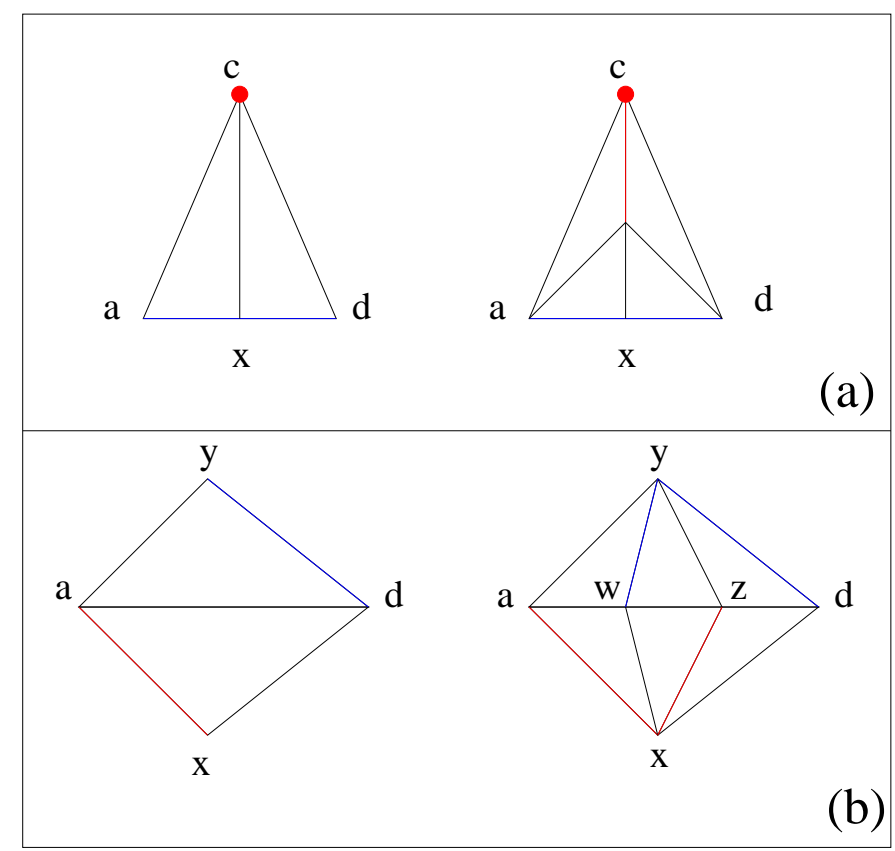

Figure 4: Simplicial subdivisions

Fig. 5 illustrates the three operations performed in a row starting from the original triangulation of a sphere as the boundary of a tetrahedron.

Now, we can triangulate $D^{3}$ as the cone over a sufficiently fine triangulation of $S^{2}$ obtained using Lemma 1.1. Then we can extend to the 1-skeleton of this triangulation $D^{3}$. Now we would like to use the idea explained in section 1.3 to extend to a subcomplex of the 2 -skeleton in such a way that each 3 -simplex has a face that belongs to this subcomplex. Of course, this extension will require a modification of our map on some 1-simplices of the triangulation of $D^{3}$ that are not in the triangulation of $S^{2}$. 


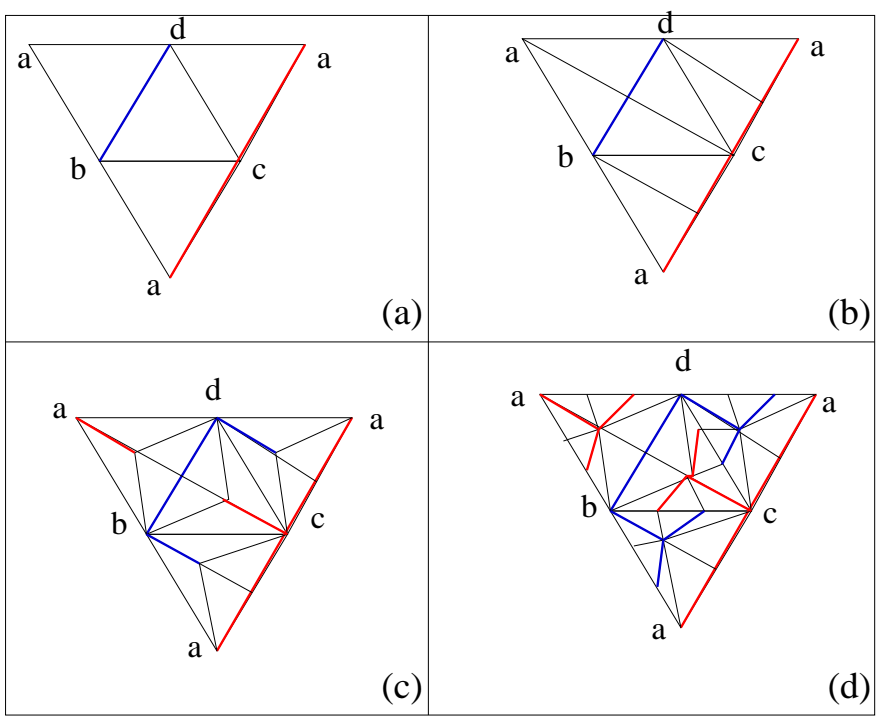

Figure 5: Constructing triangulation of $S^{2}$

Lemma 1.2 Let $S^{2}$ be triangulated as in Lemma 1.1. Let $D^{3}$ be triangulated as a cone over the chosen triangulation of $S^{2}$. Furthermore, let $f: S^{2} \longrightarrow$ $M^{n}$ be a map from $S^{2}$ to a closed Riemannian manifold $M^{n}$. Let $p$ be the image of the center of $D^{3}$. Then there exists a subcomplex $K$ of a 2-skeleton of $D^{3}$, satisfying the property that at least one 2-face of each 3-simplex of $D^{3}$ lies in $K$ and the following additional property: For every positive integer $N$ either there exist $N$ non-trivial geodesic loops in $M^{n}$ based at $p$ of length $\leq N(2 d+\delta)$ or $f$ can be continuously extended to $K$ so that $f$ maps each 2-simplex $\sigma \in K$ to a disc generated by a 1-parameter family of loops of length $\leq(N+1)(2 d+\delta)$ based at $p$ constituting a homotopy contracting the restriction of $f$ to the boundary of $\sigma$ to a constant map.

Proof. First, let us extend to the 0, 1-skeleta of $D^{3}$ as it was described in the introduction. We will now modify the extension to the 1-skeleton as follows:

Let $T_{1}, T_{2}$ be the two trees of Lemma 1.1. Let us begin with the root of $T_{1}$ that we will denote by $\tilde{v}_{0}$. Let $\tilde{v}_{i_{1}}, i_{1}=1, \ldots, k_{1}$ be the neighboring vertices of $\tilde{v}_{0}$, where $k_{1}$ denotes their number.

Consider all the edges $\tilde{g}_{i_{1}}, i_{1}=1, \ldots, k_{1}$, that connect $\tilde{v}_{0}$ with the corresponding vertices $\tilde{v}_{i_{1}}$.

Those edges correspond in a natural way to two-simplices that are ob- 
tained by coning an edge with $\tilde{p}$. Suppose we try to extend to the 2 -simplex corresponding to $\tilde{g}_{i_{1}}$. Its boundary is mapped to a closed curve of length $\leq 2 d+\delta$. Assume that when we try to contract the boundary by the BCSP, it gets stuck at a geodesic loop $\alpha_{i_{1}}$. We will then modify an extension to the edge $\tilde{e}_{i_{1}}$ that connects the vertex $\tilde{p}$ with the vertex $\tilde{v}_{i_{1}}$, to be $\alpha_{i_{1}} * e_{i_{1}}$, where $e_{i_{1}}$ denotes the image of $\tilde{e}_{i_{1}}$ under the original extension. Let us once again consider the 2 -simplex $\left[\tilde{p}, \tilde{v}_{0}, \tilde{v}_{i_{1}}\right]$. Under the new extension its boundary is mapped to a closed curve $e_{0} *\left[v_{0}, v_{i_{1}}\right] *-e_{i_{1}} *-\alpha_{i_{1}}$ of length $\leq 4 d+\delta$, (see fig. $6($ a)). This curve is contractible as a loop over the curves of length $\leq 4 d+\delta$. Also, note that $e_{0} *\left[v_{0}, v_{i_{1}}\right]$ is path homotopic to $\alpha_{i_{1}} * e_{i_{1}}$ via curves of length $\leq 5 d+3 \delta$. See fig. 6 (b) below. Indeed, one can see that $e_{0} *\left[v_{0}, v_{i_{1}}\right]$ is path-homotopic to $\alpha_{i_{1}} *-\alpha_{i_{1}} * e_{0} *\left[v_{0}, v_{i_{1}}\right]$ over the curves of length $\leq 5 d+3 \delta$. The above curve is, in its turn, path-homotopic to the curve $\alpha_{i_{1}} * e_{i_{1}} *\left[v_{i_{1}}, v_{0}\right] *-e_{0} * e_{0} *\left[v_{0}, v_{i_{1}}\right]$ also over the curves of length $\leq 5 d+3 \delta$, which finally is path-homotopic to $\alpha_{i_{1}} * e_{i_{1}}$.

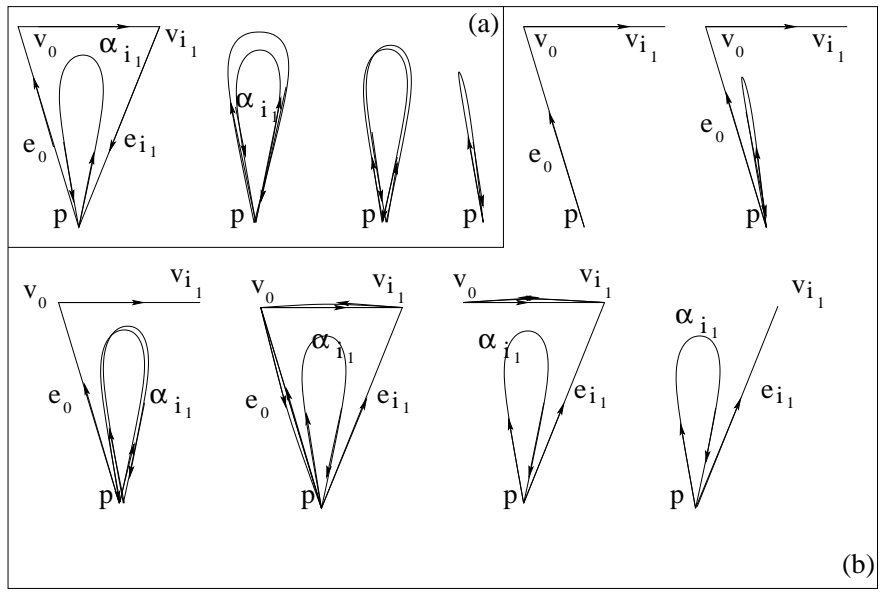

Figure 6: New extension to the 2-skeleton.

Now suppose, we have modified our extension on all 1-edges $\tilde{e}_{i_{j}}, j=$ $1, \ldots, s, i_{j} \in\left\{1, \ldots, k_{s}\right\}$, where $\tilde{e}_{i_{j}}$ are edges joining $\tilde{p}$ with vertices $\tilde{v}_{i_{j}}$, that are located at a distance $\leq s$ from $\tilde{v}_{0}$ in the tree, and $k_{s}$ denotes the number of such vertices. Suppose, in the process we were obstructed by at most $N$ distinct geodesic loops of length $\leq N(2 d+\delta)$. Suppose also that we have extended to all of the 2 -simplices of the form $\left[\tilde{p}, \tilde{v}_{i_{j-1}}, \tilde{v}_{i_{j}}\right]$, where $j=1, \ldots, s$. We now would like to extend to all of the edges of the form $\tilde{e}_{i_{s+1}}$ and all of the 2-simplices of the form $\left[\tilde{p}, \tilde{v}_{i_{s}}, \tilde{v}_{i_{s+1}}\right]$. Let us consider the image of the 
boundary. By the induction assumption, it is mapped to a curve of length $\leq(N+1)(2 d+\delta)$. Let us apply the BCSP with a fixed point $p$ to this curve. If there are no geodesic loops of length $\leq(N+1)(2 d+\delta)$, then the boundary is contractible to $p$ without the length increase and, therefore, we will not modify the image of $\tilde{e}_{i_{s+1}}$. Suppose, it does get stuck on a loop. Let us denote it $\alpha_{i_{s+1}}$. If it does get stuck on the loop of length $>N(2 d+\delta)$ then this loop is distinct from the $N$ loops we obtained during the previous steps of the induction and we will have $(N+1)$ loops of length $(N+1)(2 d+\delta)$. On the other hand, if the loop is one of the previous loops, its length must be $\leq N(2 d+\delta)$. In any of the two latter cases we will modify the extension on $\tilde{e}_{i_{s+1}}$ to be $\alpha_{i_{s+1}} * e_{i_{s+1}}$. We continue in the following manner, until we have modified an extension on all of the $\tilde{e}_{i_{j}}$ and all of the $\left[\tilde{p}, \tilde{v}_{i_{j}}, \tilde{v}_{i_{j+1}}\right]$.

Now we can repeat the same process for the tree $T_{2}$.

\section{The proof of Theorem 0.1 for loops.}

To prove Theorem 0.1 one will need the following simple lemma.

Lemma 2.1 Let $M^{n}$ be a Riemannian manifold.

Let $\gamma_{1}(t), \gamma_{2}(t)$ be two curves connecting the points $p, q \in M^{n}$ of lengths $l_{1}, l_{2}$ respectively. Consider a (not geodesic) loop $\gamma_{1} *-\gamma_{2}$ based at $p$ that is a product of $\gamma_{1}$ and $-\gamma_{2}$. Let us apply the BCSP to this loop, that keeps the point $p$ fixed. Suppose also that it converges to a geodesic loop $\alpha$ based at $p$.

Then there is a path homotopy $H_{\tau}(t), \tau \in[0,1]$, such that $H_{0}(t)=$ $\gamma_{1}(t), H_{1}(t)=\alpha * \gamma_{2}(t)$ and the length of curves during this homotopy is bounded above by $3 l_{1}+2 l_{2}$.

Proof. Let $\tilde{h}_{\tau}(t)$ denote a homotopy that connects $\gamma_{1} *-\gamma_{2}$ with a geodesic loop $\alpha$, (see fig. 2 (a) and (b)). Then below is a path homotopy between $\gamma_{1}$ and $\alpha * \gamma_{2}$ satisfying the required properties. $\gamma_{1} \longrightarrow \alpha *-\alpha * \gamma_{1} \longrightarrow$ $\alpha * \tilde{h}_{1-\tau} * \gamma_{1} \longrightarrow \alpha * \gamma_{2} *-\gamma_{1} * \gamma_{1} \longrightarrow \alpha * \gamma_{2}$, (see fig. 2 (a)-(g)). The length of curves during this homotopy is $\leq 3 l_{1}+2 l_{2}$.

This lemma generalizes a similar statement in $[R]$. Also a similar argument is used by C.B. Croke to prove Lemma 3.1 in [C].

Before stating the proof of Theorem 0.1 let us describe the idea behind it. Let us begin, as in the introduction, with the map $f: S^{2} \longrightarrow M^{n}$. Let $S^{2}$ be triangulated as in Lemma 1.1, and let us start with the modified 


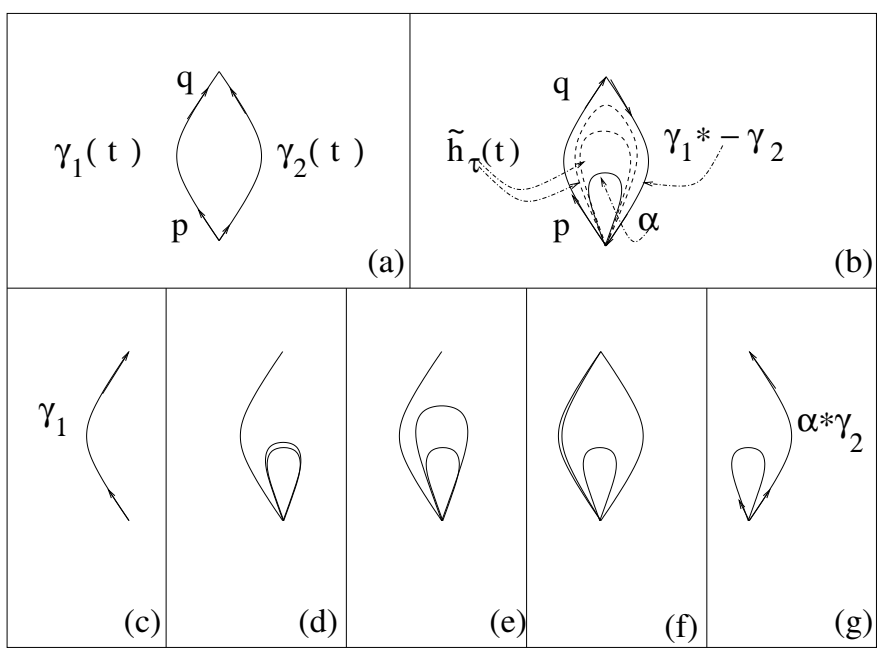

Figure 7: Illustration of the proof of Lemma 1.1.

extension from $D^{3}$ to $M^{n}$ as in Lemma 1.2 for $N=k-1$. We will now attempt to extend to the rest of the 2 -skeleton of $D^{3}$. So, let us consider a 2 -simplex $\left[\tilde{p}, \tilde{v}_{i_{1}}, \tilde{v}_{i_{2}}\right]$ to which we did not previously extend our map. From Lemma 1.2 we know that its boundary is mapped to a closed loop of length $\leq$ $k(2 d+\delta)$, or there will be $k$ distinct geodesic loops of length $\leq(k-1)(2 d+\delta)$ (including the trivial one), and we are done. Let us apply the BCSP with fixed endpoints to this loop. The process, can possibly converge to a geodesic loop $\alpha$ of length $\leq k(2 d+\delta)$, not allowing us to proceed further. If this loop happens to be a periodic geodesic, then we are done, as we can regard its iterates as distinct geodesic loops. Suppose, therefore, that this loop is not a periodic geodesic. Consider a (definitely not geodesic) loop based at $p$ that consists of going twice around the geodesic loop obtained on a previous step. When we try to contract this loop using the BCSP with fixed endpoints one of the following things can happen:

(1) This double loop contracts to a point.

(2) This double loop contracts to the same loop $\alpha$ as the original loop.

(3) This double loop contracts to a different non-trivial loop.

Case (3) results in a new "short" loop (of length at most twice the length of the original loop). In this case we can try to proceed in the above manner, i.e. by tracing the original loop three, four, etc. times and applying the BCSP to these curves hopefully obtaining new geodesic loops. Therefore, let us examine cases (1) and (2). The case (1) allows us to construct the 
desired extension. In the case (2) we use the following lemma;

Lemma 2.2 Let $M^{n}$ be a Riemannian manifold. Let $\alpha:[0,1] \longrightarrow M^{n}$ be a geodesic loop based at $p \in M^{n}$. Suppose $\alpha * \alpha$ is contractible to the loop $\alpha$ without by a path homotopy without the length increase, then the loop $\alpha$ is contractible to $p$ as a loop along the curves of length $\leq 3$ length $(\alpha)$.

Proof. Let $\alpha_{t}=\left.\alpha\right|_{[0, t]}, \gamma=\alpha * \alpha$ and $\gamma_{\tau}$ denote curves in the path

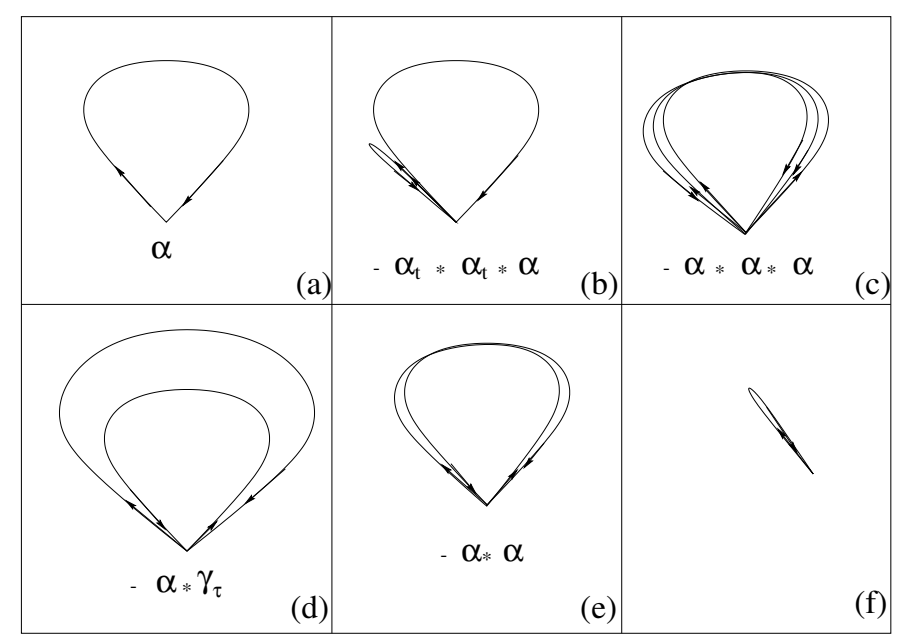

Figure 8: Double loop homotopy

homotopy that connects $\gamma$ with $\alpha$. We will now describe a path homotopy between $\alpha$ and $p$ that passes through "short" curves.

$\alpha \longrightarrow-\alpha_{t} * \alpha_{t} * \alpha \longrightarrow-\alpha * \alpha * \alpha$. The length of curves during this homotopy, obviously, does not exceed 3length( $\alpha$ ), (see fig. 8 (a)-(c)).

$-\alpha * \alpha * \alpha \longrightarrow-\alpha * \gamma_{\tau} \longrightarrow-\alpha * \alpha$. The length of curves during this homotopy is bounded by 3length $(\alpha)$, since the length of $\gamma$ does not increase during the homotopy, (see fig. 8 (d), (e)).

Finally, $-\alpha * \alpha$ can be contracted to $p$ along itself, (see fig. 8 (f)).

The above lemma is a particular and most obvious case of the following situation: Suppose, we consider a sequence of curves obtained from $\alpha(t)$ by going an integer number of times around $\alpha(t)$, i.e. going twice, three times, four times, etc., $K$ times. Then either this sequence of curves results in $K$ 
distinct "short" geodesic loops, after an application of the Birkhoff curveshortening process to each curve of the sequence, or there exists an integer $r<K$, such that a curve obtained from the original loop by tracing it $r$ times is contractible to $p$ along "short" loops based at $p$.

Lemma 2.3 Let $M^{n}$ be a Riemannian manifold, $K$ be a positive integer number, and $\alpha_{1}$ be a geodesic loop in $M^{n}$ based at $p$. Then either there exist $K$ distinct non-trivial geodesic loops, $\alpha_{1}, \ldots, \alpha_{i}, \ldots, \alpha_{K}$ of length $l_{1}, . ., l_{i}, \ldots, l_{K}$, such that $l_{i} \leq i l_{1}, 2 \leq i \leq K$, or there exist a number $r \leq K$, such that the (non-geodesic) loop $\alpha_{1}^{r}=\alpha_{1} * \ldots * \alpha_{1}$ is path-homotopic to $p$ along the curves of length $\leq(2 K-1) l_{1}$.

Proof. Suppose that there are no $K$ distinct geodesic loops based at $p$ of length $\leq K l_{1}$. Consider the first $K$ powers of $\alpha_{1}: \alpha_{1}, \alpha_{1}^{2}, \ldots, \alpha_{1}^{i}, \ldots, \alpha_{1}^{K}$. Suppose that none of these loops is path-homotopic to $p$ without the length increase. That means that, for every $i$ when we apply the BCSP to $\alpha_{1}^{i}$, it stops at a non-trivial geodesic loop $\alpha_{i}$ respectively. Our assumption implies that all these loops cannot be distinct. Therefore there exist $i \neq j \in\{1, \ldots, K\}$, such that $\alpha_{i}=\alpha_{j}$. Without any loss of generality, let us assume that $j>i$. Then $\alpha_{1}^{j-i}$ is path-homotopic to $p$ as follows: $\alpha_{1}^{j-i} \longrightarrow \alpha_{1}^{j} *-\alpha_{1}^{i} \longrightarrow \alpha_{j} *-\alpha_{i} \longrightarrow p$. The length of curves during this homotopy is bounded by $(j+i) l_{1} \leq(2 K-1) l_{1}$.

Let $M^{n}$ be a closed Riemannian manifold, satisfying the hypothesis of Theorem 0.1. Fix a point $p \in M^{n}$. Let $S^{2}$ be triangulated as in Lemma 1.1 , and $D^{3}$ be triangulated as the cone over the chosen triangulation of $S^{2}$. Let $f: S^{2} \longrightarrow M^{n}$ be a map such that its composition $f \circ H$ with the Hopf fibration $H: S^{3} \longrightarrow S^{2}$ is an element of infinite order in $\pi_{3}\left(M^{n}\right)$. Suppose that we have extended $f: S^{2} \longrightarrow M^{n}$ to the 1-skeleton of $D^{3}$ as in Lemma 1.2. Suppose that there exist at most $(k-2)$ non-trivial geodesic loops based at $p$ of length $\leq(k-1)(2 d+\delta)$. Then to extend to 2 -skeleton of $D^{3}$, we will proceed as follows: In the 2-skeleton of $D^{3}$ there will be 2-simplices of two types. The boundary of the simplices of Type I will be mapped to a curve that is contractible to $p$ in a natural way as it is described in Lemma 1.2. According to Lemma 1.1 the boundary of every 3 -simplex of $D^{3}$ contains a simplex of type I. To extend to the simplices of Type II, we will proceed as follows: let $\tilde{\sigma}_{i}^{2}=\left[\tilde{p}, \tilde{v}_{i_{1}}, \tilde{v}_{i_{2}}\right]$ be such a simplex. Its boundary is mapped to a curve passing through the point $p$ of length $\leq(k-1)(2 d+\delta)$. Let us try to contract this curve as a loop. If, indeed, it is contractible as a 
loop then we are done. If it gets stuck on one of the geodesic loops based at $p$ we can proceed as follows: let us denote this curve by $\alpha$ and let us consider its powers $\alpha^{j}, j=1, \ldots, k-1$. Let us apply the BCSP with the fixed endpoints to each of these curves. It cannot happen that BCSP ends at a different geodesic loop every time, as we have at most $(k-2)$ distinct non-trivial geodesic loops based at $p$ of length $\leq(k-1)(2 d+\delta)$. According to Lemma 2.3 the only other possibility is that some power $\alpha^{i}$ of $\alpha$ with $i \leq k$ is contractible to a point via the loops based at $p$ of length smaller than $(2 k-3) k(2 d+\delta)$. Note, however that for different $\alpha$, this power $i$ can be different. But every considered loop $\alpha \alpha^{(k-1) !}$ will be contractible via loops of length $\leq k((k-1) !+2 k-4)(2 d+\delta)$. (We can contract $\frac{k !}{i}$ copies of $\alpha^{i}$ one by one. When we contract each copy of $\alpha^{i}$ we get an extra summand $(2 k-3-i) k(2 d+\delta)$ to the length of the considered curve.) This observation will help us to finish the proof of Theorem 0.1.

Proof of Theorem 0.1 in the case, when $p=q$. The proof will be by contradiction. Let $f: S^{2} \longrightarrow M^{n}$ be a non-contractible map. Assume that $S^{2}$ is triangulated into fine simplices as in Lemma 1.1, and that $f\left(S^{3}\right)$ has induced triangulation, such that diameter of any simplex in this triangulation is smaller than $\delta$. Let $D^{3}$ be triangulated as a cone over the triangulation of $S^{2}$. Denote another copy of $S^{2}$ by $S_{*}^{2}$. Let $f_{s}: S_{*}^{2} \longrightarrow S^{2}$ denote a standard map of degree $s$. Assume $S_{*}^{2}$ is triangulated so that $f_{s}$ is a simplicial map. Let $D_{*}^{3}$ be triangulated as a cone over $S_{*}^{2}$. We can extend $f_{s}$ to a map of $D_{*}^{3}$ to $D^{3}$ by mapping $(r, \theta),\left(\theta \in S_{*}^{2}\right)$, to $\left(r, f_{s}(\theta)\right)$. We will denote this extension also by $f_{s}$. We will now attempt to extend a map $f \circ f_{s}$ from $S_{*}^{2}$ to $D_{*}^{3}$ for $s=(k-1)$ !, and as an obstruction to this extension we will obtain a map $g: S^{2} \longrightarrow M^{n}$ homotopic to $f \circ f_{s}$ that is swept-out by "short" loops in a natural way. This sweep-out can then be used to obtain the desired upper bound for $k$ short geodesic loops based at $p$ as described in the introduction.

We will first extend $f \circ f_{s}$ to the 0 -skeleton and to the 1-skeleton of $D_{*}^{3}$. The 0 -skeleton of $D_{*}^{3}$ consists of a single additional point $p_{*}$, which is the center of the disc. We will let the image of $p_{*}$ be $p$. Next, let us extend to the 1-skeleton as follows: Consider all of the edges of the form $\left[p_{*}, w_{i}\right]$ that connect the center of the disc with the vertex $w_{i}$, where $w_{i}$ is mapped to a vertex $\tilde{v}_{i}$ by $f_{s}$. There, in general, will be $s$ such vertices. All edges of this form will be mapped by $f_{s}$ to the radius of $D^{3}$ that connects the center of $D_{*}^{3} \tilde{p}$ with $\tilde{v}_{i}$.

Now we will extend to the 2-skeleton of $D_{*}^{3}$. Suppose that we have already extended $f: S^{2} \longrightarrow M^{n}$ to the part of the 2 -skeleton that includes 
one 2-simplex in the boundary of every 3 -simplex in the considered triangulation of $D^{3} \backslash \partial D^{3}$ as guaranteed by the proof of Lemma 1.2. (Recall, that these 2-simplices are called simplices of type I. Also, recall that in order to construct this extension we need first to change the previously constructed extension to some of 1 -simplices.)

Consider an arbitrary 2-simplex of $D_{*}^{3}$ of the form $\left[p_{*}, w_{j_{1}}, w_{j_{2}}\right]$, where the boundary is mapped by (the extension of) $f_{s}$ to the boundary of a simplex $\left[\tilde{p}, \tilde{v}_{i_{1}}, \tilde{v}_{i_{2}}\right]$ of type I. We can assume that $f: S^{2} \longrightarrow M^{n}$ was already extended to this 2-simplex of the considered triangulation of $D^{3}$ by the virtue of Lemma 1.2. In this case we will map $\left[p_{*}, w_{j_{1}}, w_{j_{2}}\right]$ to the simplex $\left[\tilde{p}, \tilde{v}_{i_{1}}, \tilde{v}_{i_{2}}\right]$ using (the extension of) $f_{s}$, and then use the constructed extension of $f$. There will be $s$ such 2-simplices of the considered triangulation of $D_{*}^{3}$ that are mapped to $\left[\tilde{p}, \tilde{v}_{i_{1}}, \tilde{v}_{i_{2}}\right]$.

Finally, let us the reamining 2-simplices of the considered triangulation of $D_{*}^{3}$. These are simplices of the form $\left[p_{*}, w_{j_{1}}, w_{j_{2}}\right]$, which boundary is mapped to the boundary of a simplex $\left[\tilde{p}, \tilde{v}_{i_{1}}, \tilde{v}_{i_{2}}\right]$ of type II (see the discussion preceding the proof of the theorem. Recall, that "type II" just means that the considered 2-simplex is not of type I. That is, we do not have a ready extension of $f$ to such simplices provided by Lemma 1.2.) There will be $s$ simplices like that for every pair of vertices $\tilde{v}_{i_{1}}, \tilde{v}_{i_{2}}$. Consider the images of their boundaries under the map $f \circ f_{s}: D_{*}^{3} \longrightarrow M^{n}$. The image will be the curve $\left[p, v_{i_{1}}\right] *\left[v_{i_{1}}, v_{i_{2}}\right] *\left[v_{i_{2}}, p\right]$ raised to a power $s$, i.e. traced $s$ times. (Here $v_{i_{1}}=f\left(\tilde{v}_{i_{1}}\right), v_{i_{2}}=f\left(\tilde{v}_{i_{2}}\right)$.) It is contractible with a "controlled" length increase, (length of curves in this homotopy is bounded by $k((k-1) !+2 k-$ $4)(2 d+\delta)$, see the discussion before this proof of Theorem 0.1. Otherwise there exist $k-1$ distinct non-trivial geodesic loops based at $p$, and, therefore, $k$ distinct geodesic loops based at $p$, and we are done.)

Therefore, although we cannot extend $f \circ f_{s}$ to the union of these 2simplices, we can fill the image of the boundary of their sum by a 2-disc, that will admit a desired sweep-out by "short" loops based at $p$.

Thus, we have finished working with the 2-skeleton. Now, instead of considering $f \circ f_{s}$ as a map of $S_{*}^{2}$ into $M^{n}$, let us consider this map as a map $f \circ f_{s}: \bigvee_{j} \partial \sigma_{j}^{3} \longrightarrow M^{n}$ from the wedge of the boundaries of all 3-simplices of the considered triangulation of $D_{*}^{3}$ (joined at a point $\tilde{p}$ ). Of course, the wedge of the boundaries of the 3 -simplices can be connected with $S_{*}^{2}$ by a homotopy that removes pairs of "inner" 2-simplices with opposite orientations. Vice versa, we can map $S_{*}^{2}$ into the product of the boundaries of 3-simplices by "inserting" all inner 2-simplices twice with opposite orientations. The 
inverse image of $\tilde{p} \in \bigvee_{j} \partial \sigma_{j}^{3}$ under this map is a connected 1-dimensional set. When $f \circ f_{s}$ is regarded as a map of the product of boundaries of the 3 -simplices, it comes with a natural sweep-out by loops with short images that will be described below. This sweep-out corresponds to a sweep-out of $S_{*}^{2}$ by loops with "short" images of the same length that can be used to obtain the desired upper bound for the lengths of $k$ geodesic segments as it had been explained in the introduction.

Now we are going to describe the desired sweep-out of $\bigvee_{j} \partial \sigma_{j}^{3}$ by loops with "short" images. We begin by considering an arbitrary simplex in the triangulation of $D^{3}$ denoted $\sigma_{l}^{3}$. Then $f_{s}^{-1}\left(\sigma_{l}^{3}\right)$ will be the wedge of $s 3$ simplices $D_{i}^{3}$ in the triangulation of $D_{*}^{3}$. Next consider $f \circ f_{s}: \bigvee_{i} \partial D_{i}^{3} \longrightarrow$ $M^{n}$. This map has a natural sweep-out by short loops that will be described as follows. Let us look at the image. It is constructed from a small simplex $\sigma_{i}^{2}=\left[v_{i_{1}}, v_{i_{2}}, v_{i_{3}}\right]=f\left(\tilde{\sigma}_{i}^{2}\right)$ in the following way. Firstly, one connects $p$ with each of the vertices $v_{i_{j}}, j=1,2,3$ by curves $f\left(\tilde{e}_{j}\right)$, where $\tilde{e}_{j}$ are segments connecting $\tilde{p}$ with $\tilde{v}_{i_{j}}$. We then obtain three closed curves: $e_{j} *\left[v_{i_{j}}, v_{i_{\text {jmod } 3+1}}\right] *-e_{\text {jmod } 3+1}, j=1,2,3$. By Lemma 1.2 at least one of these curves is contractible to $p$ along "short" curves, and without any loss of generality we can assume that $e_{3} *\left[v_{i_{3}}, v_{i_{1}}\right] *-e_{1}$ is such a curve. The homotopy generates a 2 -dimensional disc that we will denote $\sigma_{31}^{2}$. This disc, as well as $\sigma_{i}^{2}$ will be taken with a multiplicity $s$. The remaining two curves are both path-homotopic to $p$ along "short" curves after they have been traced $s$ times. We would like to comment that $\sigma_{i}^{2}$ is arbitrarily small and, therefore, can be treated as a point $q$, (see the Remark 2.5 below for the details. To avoid a misunderstanding note that the notation $q$ is used here and below in this section differently than in the text of Theorem 0.1 . This is justufied by the fact that here we study only lengths of geodesic loops based at $p$ and not of geodesics connecting $p$ with an arbitrary point that was denoted $q$ in the text of Theorem 0.1). We now present a sweep-out by short curves. See also fig. 10. $p \sim e_{1} *-e_{2} * \ldots * e_{1} *-e_{2}$, (fig. 10 (a)) We next use Lemma 2.1 to "replace" $e_{1}$ by $e_{3}$, (fig. 10 (b)). $e_{1} *-e_{2} * \ldots * e_{1} *-e_{2} \sim e_{3} *-e_{2} * \ldots * e_{3} *-e_{2}$. Finally, this curve is path-homotopic back to $p$, (see fig. 10 (c)).

So, we can separately sweep-out $f \circ f_{s}: f_{s}^{-1}\left(\partial \sigma_{l}^{3}\right) \longrightarrow M^{n}$ for all such 3simplices in the triangulation of $D^{3}$. Now we can combine these sweep-outs to obtain a sweep-out of the whole map.

Note that the longest loops in the sweep-out appear when we contract $e_{1} *-e_{2}$ or $e_{3} *-e_{2}$ iterated $s$ times. Thus, we obtain $L \leq\left(2 k !+4 k^{2}-8 k\right) d$, and Proposition A implies that the length of the shortest $k$ geodesic loops on $M^{n}$ (including the trivial) does not exceed $2 k L \leq\left(4 k !+8 k^{2}-16 k\right) d$. If 
$k \geq 3$, then $\left(4 k !+8 k^{2}-16 k\right) d \leq 8 k ! d$. (If $k=2$, then a better bound $4 d$ for the length of a shortest non-trivial geodesic loop based at $p$ can be obtained as a particular case of the main result of $[R])$.

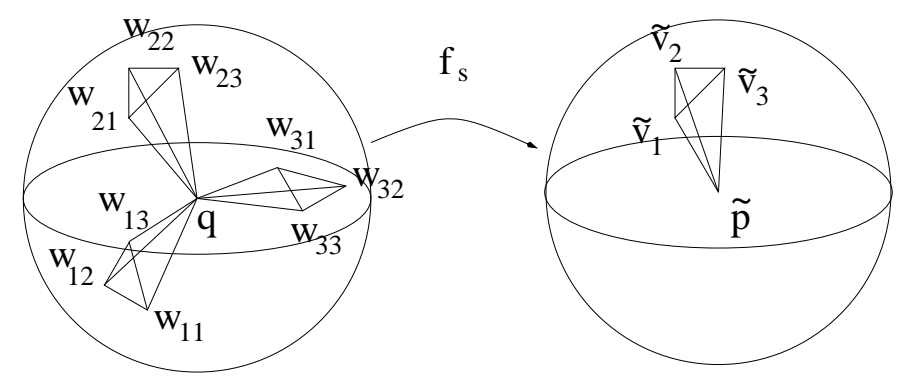

Figure 9: Extending a map $f \circ f_{s}: S^{2} \longrightarrow M^{n}$

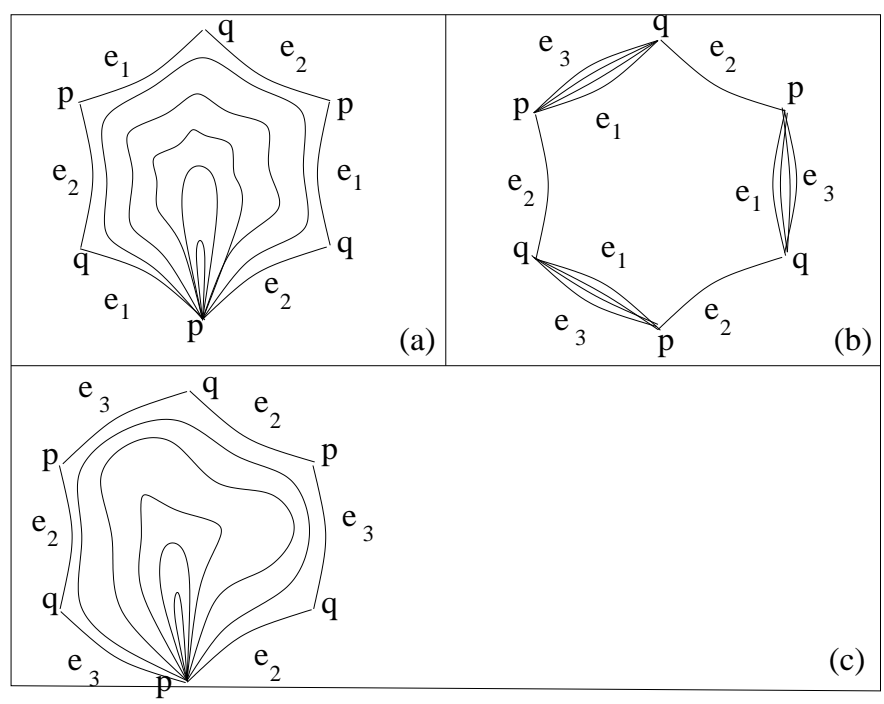

Figure 10: Sweep-out of a sphere

Remark 2.4. Note that we absolutely needed in this proof a possibility to contract the boundary of one of the three "not small" 2-simplices in the boundary of the considered 3 -simplex to a point without iterating it $s$ times. Otherwise we would not be able to obtain a desired sweep-out. This explains the necessity of Lemmae 1.2 and 1.1 . 
Remark 2.5. In this remark we will explain why the small simplex $\left[v_{i_{1}}, v_{i_{2}}, v_{i_{3}}\right]$ mentioned in the proof of Theorem 0.1 can be treated as a point. In fact, $\left[v_{i_{1}}, v_{i_{2}}, v_{i_{3}}\right]$ can be "replaced" by $q=v_{i_{2}}$, (see fig. 11). In fig. 11 we show that there is a "short" path-homotopy between two curves $e_{1} *\left[v_{i_{1}}, q\right]$ and $e_{3} *\left[v_{i_{3}}, q\right]$, (see fig. $11(\mathrm{a})$ ), that goes as follows: Firstly, note that by Lemma 2.1 there is a path homotopy passing through "short" curves between $e_{1} *\left[v_{i_{1}}, v_{i_{3}}\right]$ and $e_{3}$ during which the points $p$ and $v_{i_{3}}$ remain fixed. Let us denote the curves in this homotopy by $c_{\tau}^{1}$. Secondly, because $\left[v_{i_{1}}, v_{i_{2}}, v_{i_{3}}\right]$ can be made arbitrarily small, (in particular, its diameter can be made much smaller than convexity radius) it can be sweptout by curves of length $\leq \varepsilon(\delta)$, where $\varepsilon(\delta)$ approaches 0 as $\delta$ approaches 0 , with fixed endpoints $v_{i_{1}}$ and $v_{i_{2}}=q$. Let these curves be denoted as $c_{s}^{2}$. (See fig. $11(\mathrm{~d})$ ). Now the homotopy will go as follows, (see 11 (b)-(f)). $e_{1} *\left[v_{i_{1}}, q\right] \sim e_{1} * c_{s}^{2} \sim e_{1} *\left[v_{i_{1}}, v_{i_{3}}\right] *\left[v_{i_{3}}, q\right] \sim c_{\tau}^{1} *\left[v_{i_{3}}, q\right] \sim e_{3} *\left[v_{i_{3}}, q\right]$.

Thus, the point $q$ replaces $\left[v_{i_{1}}, v_{i_{2}}, v_{i_{3}}\right], e_{1}$ is replaced by $e_{1} *\left[v_{i_{1}}, q\right]$ and $e_{3}$ is replaced by $e_{3} *\left[v_{i_{3}}, q\right]$.

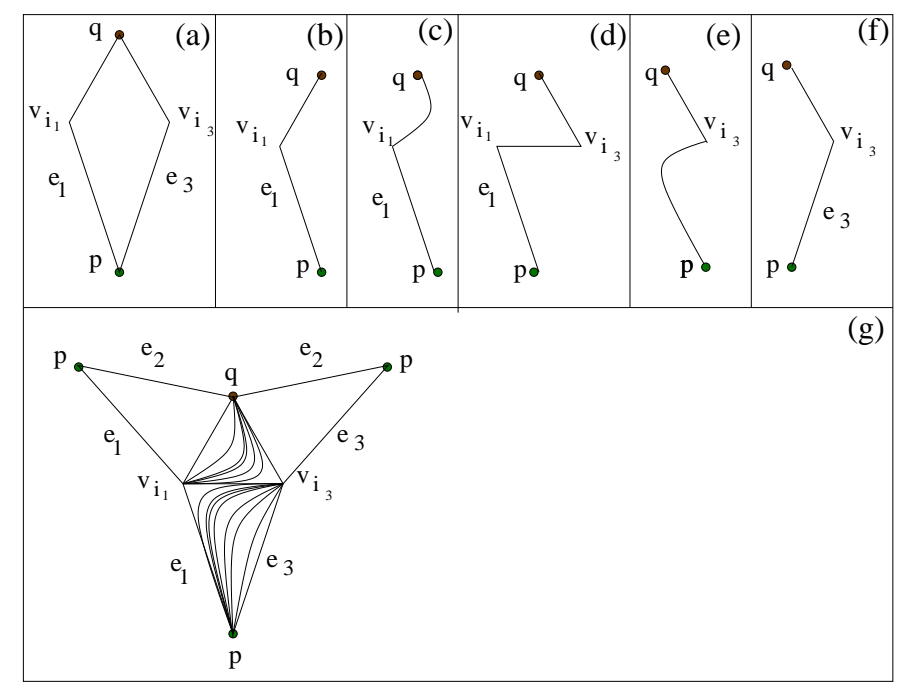

Figure 11: Small simplices can be ignored.

\section{Geodesic segments between different points.}

In the previous sections we assumed that the points $p$ and $q$ coincide, i.e. we were interested in geodesic loops based at $p$. In this section we outline 
how this proof can be generalized for the case, when $p$ and $q$ are allowed to be distinct points. The main problem is with the first step of the extension process: When we contract a loop based at $p$ using a loop shortening process, we are either able to contract it all the way to a point, or get stuck at a geodesic loop, not at a geodesic between $p$ and $q$. The remedy can be found at our paper [NR1]. Namely, we replace the Birkhoff curve-shortening process for loop by another curve shortening process described in the proof of Lemma 1 in [NR1]. It works as follows: Attach to a considered loop a fixed minimal geodesic $\sigma$ between $p$ and $q$. We obtain a path between $p$ and $q$. Now apply a version of the Birkhoff curve-shortening process for curves with fixed endpoints to this path. The result will be a geodesic between $p$ and $q$. If for some $S$ there is only one geodesic between $p$ and $q$ of length $\leq S$, then this process will be continuous with respect to initial loop based at $p$, when we consider only loops of length $\leq S-\operatorname{dist}(p, q)$. Further, assume that $\lambda_{1}$ and $\lambda_{2}$ are loops based at $p$. Assume that when we apply this process to $\lambda_{1}$ and $\lambda_{2}$ we get stuck at the same geodesic $\mu$ between $p$ and $q$. Then there is the following path homotopy between $\lambda_{1}$ and $\lambda_{2}$ that passes through loops of length $\leq \max \left\{\right.$ length $\left(\lambda_{1}\right)$, length $\left.\left(\lambda_{2}\right)\right\}+2$ dist $(p, q)$ (compare with [NR2]): Choose a minimizing geodersic $\sigma$ between $p$ and $q$. Now proceed as follows :

$$
\lambda_{1} \longrightarrow \lambda_{1} * \sigma * \sigma^{-1} \longrightarrow \mu * \sigma^{-1} \longrightarrow \lambda_{2} * \sigma * \sigma^{-1} \longrightarrow \lambda_{2}
$$

Here arrows denote obvious path homotopies between loops based at $p$.

A modification of same idea can be used in the proof of an analog of Lemma 1.2: If $\lambda_{1}, \lambda_{2}$ are two paths between $p$ and $x, \sigma$ is a minimal geodesic between $p$ and $q$, we can consider path $\lambda_{1} * \lambda_{2}^{-1} * \sigma$ between $p$ and $q$. Apply the Birkhoff curve shortening process with fixed endpoints to this path. Assume that the process stops at a geodesic $\tau$ between $p$ and $q$. Of course, the length of $\tau$ does not exceed the sum of lengths of $\lambda_{1}, \lambda_{2}$ and $\sigma$. Consider now a new path $\lambda_{2}^{*}=\tau * \sigma^{-1} * \lambda_{2}$. Now $\lambda_{1} * \lambda_{2}^{*-1} * \sigma$ can be contracted to $\sigma$ by a length non-increasing path homotopy. Therefore $\lambda_{1} * \lambda_{2}^{*-1}$ can be contracted to a point by a path homotopy that increases the length by not more than $2 d$. This observation leads to an analog of Lemma 1.2 where we conclude either the existence of $N+1$ distinct geodesics between $p$ and $q$ of length $\leq(4 N+1)(d+\delta)$ or the extendability of $f$ on each simplex $\sigma \in K$ so that the extension is generated by a 1-parametric family of loops of length $\leq 4 N(d+\delta)$. Note that this upper bound is approximately twice the bound in the original estimate. As the result the upper bound that we obtain when 
$p$ and $q$ can be different is asymptotically twice the upper bound in the loop case $((8 k !+o(k !)) d$ versus $(4 k !+o(k !)) d)$.

This idea can also be used to modify Lemma 2.3 as follows: Now we do not attempt to contract powers of $\alpha$ to a point by a length non-increasing path homotopy as in its original version. Instead we attach $\sigma * \sigma^{-1}$ to $\alpha^{i}$ at its endpoint and apply the Birkhoff curve shortening process for curves with fixed endpoints to $\alpha^{i} * \sigma$. Assume that this process converges to a geodesic $\tau_{i}$. Then, if $\tau_{i}=\tau_{j}$, then $\alpha^{i}$ is homotopic to $\alpha^{j}$ via $\tau_{i} * \sigma^{-1}$ as above, and $\alpha^{|i-j|}$ is contractible via curves of length not exceeding $(2 \max \{i, j\}-\min \{i, j\})$ length $(\alpha)+2 \operatorname{dist}(p, q)$, and an analog of Lemma 2.3 easily follows.

Acknowledgments. We would like to thank Misha Gromov for a very useful discussion of the problem. In particular, he gave an advice to the first author to look at iterates of an obstructing geodesic loop. This advice was amply used in our proof. This paper was partially written during both authors visit of the Max-Planck Institute for Mathematics at Bonn. The authors would like to thank the Max-Planck Institute for its kind hospitality. The first author gratefully acknowledges a partial support of this research by his NSERC Discovery Grant and NSF Grants DMS-0405954 and DMS-0706803. The second author gratefully acknowledges a partial support of this research by her Natural Sciences and Engineering Research Council (NSERC) University Faculty Award and Discovery Grant and a partial support of this research by the NSF Grant DMS-0604113.

\section{References}

[BCK] F. BalachefF, C. Croke, M. Katz, "A Zoll counterexample to a geodesic length conjecture", to appear in Geom. Funct. Anal. (GAFA).

[BZ] Ju. Burago, V. Zalgaller, Geometric Inequalities, Springer-Verlag, Berlin, 1988.

[C] C. B. Croke, Area and the length of the shortest closed geodesic, J. Diff. Geom. 27 (1988), 1-21.

[CK] C. B. Croke, M. Katz, Universal volume bounds in Riemannian manifolds, Surveys in differential geometry. Vol. VIII (Boston, MA 2002), 109-137, Surv. Diff. Geom. VIII, Int. Press. Somerville, MA, 2003.

[FHT] Y. Felix, S. Halperin, J. C. Thomas, Rational Homotopy Theory, Springer, 2001.

[FK] S. Frankel, M. Katz, The Morse landscape of a Riemannian disk, Ann. Inst. Fourier (Grenoble), 43(1993), 503-507. 
[G] M. Gromov, Filling Riemannian manifolds, J. Diff. Geom. 18 (1083), $1-147$.

[Kl] W. Klingenberg, Lectures on closed geodesics, Springer, 1978.

[NR0] A. Nabutovsky, R. Rotman, Lengths of geodesics between two points on a Riemannian manifold, Electron. Res. Announc. (ERA) Amer. Math. Soc. 13(2007), 13-20.

[NR1] A. Nabutovsky, R. Rotman, The length of the second shortest geodesic, to appear in Comment. Math. Helv.

[NR2] A. Nabutovsky, R. Rotman, Lengths of geodesics on a two-sphere, to appear in American J. Math.

[R] R. Rotman, The length of a shortest geodesic loop at a point, J. Differential Geom. 78(2008), no. 3, 497-519.

[S] S. Sabourau, Global and local volume bounds and the shortest geodesic loops, Commun. Anal. Geom. 12 (2004), 1039-1053.

[Sch] A. Schwarz, Geodesic arcs on Riemannian manifolds, Uspekhi Math. Nauk (translated as "Russian Math. Surveys"), 13(1958), 181-183.
A. Nabutovsky and R. Rotman
Department of Mathematics
Department of Mathematics
University of Toronto
Penn State University
Toronto, Ontario M5S 2E4
University Park, PA 16802
Canada
USA
e-mail: alex@math.toronto.edu
nabutov@math.psu.edu
e-mail:rina@math.toronto.edu
rotman@math.psu.edu 